\title{
Welcoming Healthcare Into Our Virtual Meeting Rooms: Perspective and Perseverance in Pediatric Rheumatology
}

\author{
Mary M. Eckert ${ }^{1} \cdot$ Natalie L. Rosenwasser $^{1} \cdot$ Stephen C. Wong ${ }^{1}$ \\ Accepted: 16 August 2021 / Published online: 4 October 2021 \\ (c) The Author(s), under exclusive licence to Springer Science+Business Media, LLC, part of Springer Nature 2021
}

\begin{abstract}
Purpose of Review We highlight practice changes adopted to increased use of telemedicine, look at precision, and accuracy in using a virtual visit to evaluate and treat rheumatic disease, and describe our shift in engaging patients and their families in supporting our research aims.

Recent Findings Telemedicine visits increased substantially with the start of the SARS-CoV-2 pandemic. With this change came the need for significant advances to our telemedicine practices to allow for quality patient visits and continued research collection.

Summary Telemedicine will continue to be an area of increasing importance and has been found to be especially useful for regions like ours which cover many patients in remote areas across Washington, Wyoming, Idaho, Montana, and Alaska. Through the development of new techniques and the use of new technologies, we have been able to improve both the visit quality for patients and our ability to collect research data.
\end{abstract}

Keywords Telemedicine $\cdot$ Pediatric $\cdot$ Rheumatology $\cdot$ COVID $\cdot$ Research

\section{Introduction}

With the rise of high-speed internet and portable devices came the advent of a new medium of healthcare: telemedicine. Since its inception, the field of telemedicine has evolved rapidly; however, it is clear that this past year has driven changes in telemedicine more than ever before. Acceleration in the adoption and use of telemedicine emerged after a national emergency was declared on March 13, 2020, due to the SARS-CoV-2 pandemic [1]. Hospitals and healthcare providers faced a global pandemic and grappled with the need to provide care to patients while working within national guidelines to control the spread of the virus.

Mary M. Eckert, Natalie L. Rosenwasser and Stephen C. Wong contributed equally.

This article is part of the Topical Collection on Technology and Pediatrics

Mary M. Eckert

mary.eckert@seattlechildrens.org

1 Division of Rheumatology, Department of Pediatrics, University of Washington/Seattle Children's Hospital, 4800 Sand Point Way NE, Seattle, WA 98105, USA
The Centers for Medicare and Medicaid Services (CMS) temporarily waived regulatory waivers and created new rules including granting licensed providers access to serv-

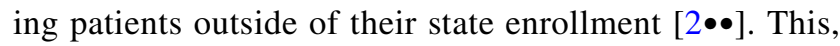
combined with relaxed telemedicine reimbursement policies, allowed for rapid adoption across many hospital and clinical

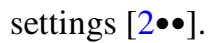

Given the rapid spread of the virus, on April 7, 2020, the CMS made recommendations to postpone any non-essential medical care, which allowed for decisions surrounding nonessential care be made "at the local level by the clinician, patient, hospital, and local health departments" [3, 4]. Due to the emergency measures hospitals faced, there was a strong need to preserve space for patients with acute SARS-CoV-2 infection, decrease patient throughput accessing non-emergent healthcare, along with protecting healthcare workers and patients facing limited supplies of personal protective equipment. It became clear that a new process and need for transition of in-person visits to telemedicine needed to be expedited to meet the needs of patients.

Within the field of rheumatology, the American College of Rheumatology (ACR) responded with a position statement supporting the role of telemedicine to increase access and improve care for patients with rheumatic illness yet 
highlighted that telemedicine should not replace face-to-face assessments at appropriate intervals. They did acknowledge the limitations of relevant technologies in telemedicine delivery, but ultimately supported its use with guidelines $[5 \bullet \bullet]$.

In efforts to summarize and streamline resources in pediatric rheumatology, Shenoi and Hayward et al. summarized a table of valuable information to aid other providers and insti-

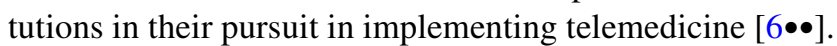
This included highlighted telemedicine practice guidelines, practical and logistical tips including technical platform and equipment considerations, telemedicine scripts and dot phrases for use in the electronic medical record, a suggested examination schedule, and aids in patient and family visit preparation. Resources on how to perform a virtual pediatric musculoskeletal exam, the V-pGALS (pediatric Gait, Arms, Legs, and Spine) assessment, were proposed as a variation to the in-person pGALS already implemented in in-person visits.

In this review, we highlight practice changes adopted to increased use of telemedicine, briefly discuss the precision and accuracy in using a virtual visit to evaluate and treat rheumatic disease and describe our shift in engaging patients and their families in supporting our research aims.

\section{Telemedicine: Process Changes in Pediatric Rheumatology}

As legal and formal processes for telemedicine conversion rolled out in real time, many limitations were encountered. Not all patients had access to reliable internet or an electronic device that could support telemedicine software. Families and providers struggled with the need to troubleshoot unexpected technical roadblocks in real time while providers grappled with providing meaningful care in those situations. Delivering equitable care to patients with language service needs, and to those in remote regions, also had to be taken into account. To deliver high-quality care, our hospital found ways of creating new processes to meet these needs while providers found novel ways of working alongside primary care providers, medical translators, medical assistants, and nurses to help facilitate visits.

Postponing care was not an option for many patients seen at Seattle Children's Hospital ( $\mathrm{SCH})$. $\mathrm{SCH}$ providers cover the WWAMI region. The WWAMI region encompasses the states of Washington, Wyoming, Alaska, Montana, and Idaho. Given the temporary expansion of telehealth services during the SARS-CoV-2 pandemic, Federally Qualified Health Centers (FQHCs) and Rural Health Clinics (RHCs) allowed for an extension of providers not licensed in all

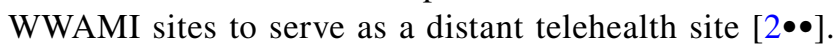
Pediatric rheumatologists at $\mathrm{SCH}$ cover the vast amount of pediatric rheumatology care in Alaska, Montana, and Washington, as well as other surrounding states such as Idaho and Oregon. Given the vast geographic coverage, an enormous burden is placed on families to seek and establish continuity of care for children with rheumatic disease.

Children with rheumatic conditions have chronic diseases which require continued evaluation and treatment. In the division of pediatric rheumatology, it was quickly realized that ongoing need for care for our large catchment area provided us with a pressing opportunity. As a result, visits were not postponed or cancelled but instead quickly converted to telemedicine, when appropriate. Appointments were reviewed weeks ahead of time by the provider they were scheduled with, tiered, and converted based on the urgency and need for in-person visits. The division met regularly to discuss hospital policies and protocols and the criteria for who should be seen in person shifted as provider comfort with telemedicine increased. Conceptualizing the new reality of what telemedicine visits would entail took time, practice, and a new level of comfort and confidence on both the provider and patient fronts. Our division became even more aware of the patient's distance from our clinic sites and used the activity of a patient's disease and disease type in the determination of the need for follow-up in person or virtually.

\section{Telemedicine: Adopting Technology and V-pGALS}

Telemedicine visits have been difficult to implement in the pediatric rheumatology clinic since rheumatologists place a high value on a thorough physical exam. Without the ability to perform an in-person physical exam, a diagnosis can be easily missed. Rheumatologists often assess for joint swelling, warmth, tenderness, symmetry, as well as muscle strength or atrophy, and clearly characteristic cutaneous rashes. Given the concern for a less than optimal examination, the reluctance of using telemedicine visits in pediatric rheumatology practice is clear.

In rheumatology, accurately assessing joints for active inflammation without the ability to examine the joints in person was a concern many providers shared. Historically, the Gait, Arms, Legs, Spine (GALS) exam has been shown to have high sensitivity in detecting significant musculoskeletal abnormalities [7]. The pediatric equivalent of the GALS, pGALS, was adapted and validated for use for children [8]. A proposed variation of the pGALS for virtual use, the V-pGALS, was implemented into our telemedicine

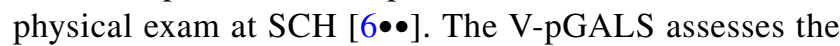
range of motion of all joints in the arms, knees, ankles, feet, and spine and assesses gait as well. Caregivers are also asked to feel for warmth, swelling, or crepitus of the 
knees to improve sensitivity of the knee exam. Lastly, temporomandibular joints are visualized for deviation with range of motion, and a maximal oral aperture is assessed and evaluated using a 3-finger wide test (a child is asked to place three side-by-side fingers into their mouth vertically). These joints are the most likely joints to present with arthritis in Juvenile Idiopathic Arthritis (JIA), the most common disease seen in pediatric rheumatology. Although the V-pGALS may be viewed as a less sensitive exam compared with an in-person physical exam, valuable information can be obtained during a virtual visit that may be concerning for and consistent with active inflammatory arthritis.

The adoption of telemedicine in pediatric rheumatology at $\mathrm{SCH}$ was slow prior to the SARS-CoV-2 pandemic. In 2019 , at $\mathrm{SCH}$, a total of 14 telehealth visits were completed out of 4451 total outpatient clinic visits leading to a disproportionately low telehealth visit rate of $0.31 \%$. This statistic is likely consistent with similar rates seen over recent years. As the SARS-CoV-2 pandemic limited in-person access to medical care and providers struggled with providing medical care to those with chronic conditions, a drastic shift in conversion from in-person to telemedicine was noted. In 2020,1260 out of a total of 4497 clinic visits $(28.0 \%)$ were telemedicine visits, which also included the first quarter of 2020 (pre-pandemic data). This shift in high telemedicine utilization continued into the first quarter of 2021 with 140 out of 354 patients provided with virtual medical care (39.5\%) marking an era of increased utilization that will likely persist into the future. At $\mathrm{SCH}$, providers have made switches from in-person to telemedicine fluid in the event that families have an emergency or simply cannot make it to our site for a variety of reasons. We have also adopted one clinic a month, per provider to help streamline support staff availability and continued telemedicine access to patients marking this new practice shift going forward.

The question of how our virtual exams fared with traditional in-person visits was something we wanted to explore. Given the shift to utilizing the V-pGALS exam, we aimed to determine whether pediatric rheumatologists at $\mathrm{SCH}$ were scoring actively inflamed joints accurately for patients with JIA. We analyzed 38 charts of children with JIA in the first quarter of 2021. To determine if we were accurately scoring (and not over or underscoring active joints) active inflammatory arthritis via telemedicine, an average joint count was assessed using a chart review at a telemedicine and subsequent in-person visit. The mean active joint count using V-pGALS was 0.38 compared to 0.97 for in-person physical examinations performed in outpatient clinic visits. There was no significant difference in the paired means $(p=0.20)$ though telehealth exams trended towards underscoring active joints. Overall, it appears that the V-pGALS is a reasonable alternative for identifying active arthritis.
Most V-pGALS examinations noted 0 active and questionable joints at $71 \%$ (27/38), while the number of days in between visits averaged 147 days (4.7 months). This data suggests that while access to in-person visits has increased in the first quarter of 2021, pediatric rheumatologists preferentially chose to see children with mild or inactive disease via telemedicine and continued to stratify those with more severe or active arthritis to be seen in the clinic.

\section{Telemedicine: Incorporating Research}

With the need to decrease non-essential personnel entering the hospital, research coordinators and much of non-urgent research-related visits were halted in mid-March of 2020 as SARS-CoV-2 cases increased in Washington State. Clinical, translational, and bench research along with clinical trial work were all affected by the SARS-CoV-2 restrictions. This included work at $\mathrm{SCH}$ along with work being completed within our division in pediatric rheumatology. While our primary concern was to provide patient care, our ability to continue collecting data and enrolling participants in research studies was a factor to consider when transitioning patients from in-person to telemedicine visits.

In the transition to telemedicine, the process by which we would continue to administer surveys, previously completed in person, and collect data and biospecimens was carefully considered. Additionally, it was important to ensure that the data collected from provider visits was valid and reliable. The focus was on collecting quality data, to ensure that accurate observations could be made when reflecting back on this time period.

Initially during the telemedicine transition rollout, we determined that the best way to collect patient reported outcomes (PRO) was to have a clinical research coordinator (CRC) ask research-related questions verbally to participants, either via the telemedicine visit or via a telephone call. Unfortunately, this process was laborious and given the large number of participants could not carry forward. On average, each encounter with a research participant was estimated at an average of $30 \mathrm{~min}$. Time constraints of our CRC's time along with study constraints, the need to obtain research-related information, usually within $48 \mathrm{~h}$, made gathering this information difficult. Instead, a prioritized agenda was constructed with an aim to collect at least two of the most critical PRO questions from every telemedicine visit, as set forth by the study. To accomplish this, electronic reminders and scripts were sent from CRCs to providers at each clinic visit, including patient names and research questions to be asked during telemedicine appointments. If the information was not obtained during the visit, CRCs would call the patient or family to obtain this information after their appointment. These changes in data collection and 
prioritizing research associated questions made for a much more manageable workflow, while still ensuring data could be collected from all participants enrolled.

We also needed to address how data and information from the provider's visit notes was collected for research purposes. Just as our providers could not collect all of the commonly used disease measurements from an in-person visit in their telemedicine visits, CRCs were unable to use this information for our research agendas. Variables such as active joint count were modified to active joint count and questionable joint count (as determining true arthritis activity virtually could be challenging). Items like serositis, splenomegaly, and lymphadenopathy could not be assessed without an in-person physician examination. As research data collection could not be modified in some instances, many items had to be represented as "unknown" and the data from these visits lacked.

With more provider experience and practice in using telemedicine, continued discussion within the division, and implementation of new carefully decided upon processes, we improved our data collection and telemedicine capabilities for both clinic and research. For example, by outlining and implementing the V-pGALS assessment, providers in our division set a strong precedence for the continued use of telemedicine in a way that would allow both clinic visits and research collection to continue to grow and thrive [6••].

Over time, some study questionnaires were converted to an electronic online format in attempts to email them to our patients after completing a telemedicine visit. Unfortunately, this format proved that CRC involvement was still paramount since using this model yielded lower survey completion rates. CRCs continued their involvement in speaking with patients and their family before or after the telemedicine visit as a reminder that surveys would be sent and to help encourage survey completion. CRCs would send the survey during their visit and in some instances would follow up as needed.

In the future, online questionnaires will be e-mailed to enrolled participants prior to their visit as a reminder to help with completion rates. This implementation could increase data yield and help patients and their families who may lack the time or mental and emotional energy at the time of their visit.

\section{Conclusion}

The SARS-CoV-2 pandemic has highlighted the need for increased modalities to deliver care to patients. With time, hospitals, clinics, providers, ancillary staff, and patients will continue to cater to a population who greatly benefits from telemedicine. Given our geographic distribution, the ability to improve access to continuous care during the most challenging times has become our reality. We are privileged to live in a time where the technological capability has allowed for rapid implementation and anticipate continued improvement going forward.

As we continue to integrate telemedicine into our practice going forward, we have become creative in utilizing telemedicine to serve our patients in Montana. We have combined a rheumatology telemedicine visit at $\mathrm{SCH}$ with an in-person visit at Community Medical Center (CMC) in Missoula staffed by a rheumatology-trained orthopedic physician assistant as a "telepresenter" to aid us in performing thorough joint and musculoskeletal exams. This monthly clinic has allowed for more robust continuity of care for this population of patients. With time, we anticipate more creative collaborations to continue to serve patients within our geographic catchment.

At $\mathrm{SCH}$, our pediatric rheumatologists were able to demonstrate that a significant number of patients could be seen virtually, especially for patients for whom travel to the clinic is a barrier to care. We have shown that a large proportion of our patients can be seen via telemedicine and that using the V-pGALS assessment can be adapted during these visits as an acceptable way to assess children with rheumatic disease in children who have mild or stable disease.

We have learned that research can be integrated into virtual visits, though research implementation will continue to evolve as study protocols adjust in light of providing care remotely. As telemedicine utilization continues, it will be important to consider study protocol implementation to incorporate telemedicine practice earlier in grant procurement and protocol development.

This critical time in history has made principal investigators (PIs) more aware of the need to build online and paperless capabilities for research agendas and patient consent. This realization will aid in future consent of families who are interested in participating but who have previously not been able to participate as no formalized process was available for electronic consent. Given the increasing prevalence of telemedicine utilization, the process by which participants will be approached will be important in ensuring an equitable opportunity for every patient qualified to participate in research. This will aid in increasing diversity and representation of patient involvement in research and minimize geographic boundaries in participation.

Acknowledgements Special thanks to Eric Allenspach, MD, PhD; Sarah Baxter, MD, PhD; Sriharsha Cherukumilli Grevich, MD, MAS; Kristen Hayward, MD, MS; Kabita Nanda, MD; Susan Shenoi, MB BS, MS; Anne Stevens, MD, PhD; Yongdong Zhao, MD, PhD; Joshua Scheck, BA, BS for providing collaborative data.

Special thanks to Daniel Rosenwasser for assisting in draft preparations. 


\section{Declarations}

Conflict of Interest The authors declare no competing interests.

\section{References}

Papers of particular interest, published recently, have

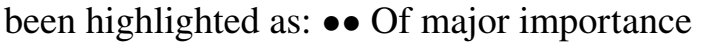

1. Centers for Medicare \& Medicaid Services, Current Emergencies, 2020, https://www.cms.gov/About-CMS/Agency-Infor mation/Emergency/EPRO/Current-Emergencies/Current-Emerg encies-page. Accessed 28 June 2021.

2.• Centers for Medicare \& Medicaid Services, COVID-19 emergency declaration blanket waivers for health care providers, https://www.cms.gov/files/document/covid-19-emergency-decla ration-waivers.pdf. Accessed 28 June 2021. (The waivers and actions the US administration took that added flexibility to help healthcare providers to contain the spread of COVID-19 disease.)

3. Centers for Medicare \& Medicaid Services, CMS releases recommendations on adult elective surgeries, non-essential medical, surgical, and dental procedures during COVID-19 response, https://www.cms.gov/newsroom/press-releases/cms-releasesrecommendations-adult-elective-surgeries-non-essential-medic al-surgical-and-dental. Accessed 28 June 2021.
4. Centers for Medicare \& Medicaid Services, Non-emergent, elective medical services, and treatment recommendations, https:// www.cms.gov/files/document/cms-non-emergent-elective-medic al-recommendations.pdf. Accessed 28 June 2021.

5.•• American College of Rheumatology, ACR releases position statement on telemedicine, Jun 29 2020. https://www.rheumatolo gy.org/About-Us/Newsroom/Press-Releases/ID/1087. Accessed 28 June 2021 . Important statement supporting the role of telemedicine as a tool in medicine.

6.•• Shenoi S, Hayward K, Curran ML, et al. Telemedicine in pediatric rheumatology: this is the time for the community to embrace a new way of clinical practice. Pediatr Rheumatol. 2020;18:85. https://doi.org/10.1186/s12969-020-00476-z. Important new rheumatology assessment tool developed for increasing telemedicine use.

7. Doherty M, Dacre J, Dieppe P, Snaith M. The 'GALS' locomotor screen. Ann Rheum Dis. 1992;51(10):1165-9.

8. Foster HE, Kay LJ, Friswell M, Coady D, Myers A. Musculoskeletal screening examination (pGALS) for school-age children based on the adult GALS screen. Arthritis Rheum. 2006;55(5):709-16. https://doi.org/10.1002/art.22230 (Erratum in: Arthritis Rheum. 2006 Dec 15;55(6):981. PMID: 17013854).

Publisher's Note Springer Nature remains neutral with regard to jurisdictional claims in published maps and institutional affiliations. 